\title{
ON THE NACHBIN TOPOLOGY IN SPACES OF HOLOMORPHIC FUNCTIONS
}

\author{
BY JORGE MUJICA
}

Communicated by Hans Weinberger March 27, 1975

1. Introduction. $H(U)$ denotes the vector space of all holomorphic functions on an open subset $U$ of a complex Banach space $E$. In this note we announce results concerning the Nachbin topology $\tau_{\omega}$ in $H(U) . \tau_{\omega}$ is useful in the study of holomorphic continuation; see Dineen [5], [7] and Matos [8]. We recall the definition of $\tau_{\omega}$; see Nachbin [10]. A seminorm $p$ on $H(U)$ is said to be ported by a compact subset $K$ of $U$ if for each open set $V$, with $K \subset V \subset U$, there exists $c(V)>0$ such that $p(f) \leqslant c(V) \sup _{x \in V}|f(x)|$ for all $f \in H(U)$. The locally convex topology $\tau_{\omega}$ is defined by all such seminorms. To study $\left(H(U), \tau_{\omega}\right)$ we consider the vector spaces of holomorphic germs $H(K)$ with $K \subset U$ compact. We endow each $H(K)$ with the inductive topology given by

$$
H(K)=\underset{\epsilon>0}{\lim _{\longrightarrow}} H^{\infty}\left(K_{\epsilon}\right),
$$

where $K_{\epsilon}=\{x \in E$ : $\operatorname{dist}(x, K)<\epsilon\}$ and $H^{\infty}\left(K_{\epsilon}\right)$ denotes the Banach space of all bounded holomorphic functions on $K$, with the sup norm.

$2 .^{1}$ Completeness of $\left(H(U), \tau_{\omega}\right)$. The following theorem answers a question raised by Nachbin [11].

THEOREM 1. $\left(H(U), \tau_{\omega}\right)$ is always complete.

Earlier partial results were given by Dineen [6], Chae [3] and Aron [2] for $U$ "nice". We give an indication of the proof of Theorem 1. For each compact $K \subset U$, let $M^{K}$ denote the image of the canonical mapping $H(U) \rightarrow H(K)$. After identifying $H^{\infty}\left(K_{\epsilon}\right)$ with its image in $H(K)$, we define:

$$
\begin{aligned}
M_{\tilde{\epsilon}}^{K} & =M^{K} \cap H^{\infty}\left(K_{\epsilon}\right), \\
\widetilde{M}_{\epsilon}^{K} & =\text { closure of } M_{\epsilon}^{K} \text { in } H^{\infty}\left(K_{\epsilon}\right), \\
\widetilde{M}^{K} & =\bigcup_{\epsilon>0} \widetilde{M}_{\epsilon}^{K} .
\end{aligned}
$$

In a diagram we have

AMS (MOS) subject classifications (1970). Primary 46E10, 46E25; Secondary 32D10.

Key words and phrases. Nachbin topology, holomorphic germ, inductive limit, projective limit, multiplicatively locally convex algebra, spectrum, envelope of holomorphy.

1 The results in $\$ 2$ of this note are taken from the author's doctoral dissertation at the University of Rochester, written under the supervision of Professor Leopoldo Nachbin. 


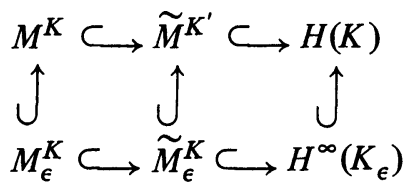

$\widetilde{M}_{\epsilon}^{K}$ is the completion of the vector subspace $M_{\epsilon}^{K}$ of the Banach space $H^{\infty}\left(K_{\epsilon}\right)$. We endow $M^{K}$ and $\widetilde{M}^{K}$ with the inductive topologies coming from

$$
M^{K}=\underset{\epsilon>0}{\stackrel{\lim }{\longrightarrow}} M_{\epsilon}^{K}, \quad \widetilde{M}^{K}=\underset{\epsilon>0}{\stackrel{\lim }{\longrightarrow}} \widetilde{M}_{\epsilon}^{K} .
$$

Theorem 1 follows from Lemmas 1 and 2, below.

Lemma 1. $\tilde{M}^{K}$ is the completion of $M^{k}$.

LEMMA 2. $\left(H(U), \tau_{\omega}\right)=\lim _{K \subset U} M^{K}=\lim _{K \subset U} \widetilde{M}^{K}$.

3. Multiplicative local convexity of $\left(H(U), \tau_{\omega}\right)$. The following theorem answers a question raised by Matos [8].

THEOREM 2. $\left(H(U), \tau_{\omega}\right)$ is a multiplicatively locally convex algebra, i.e. $\tau_{\omega}$ is defined by the continuous seminorms $p$ such that, for all $f, g \in H(U)$,

$$
p(f g) \leqslant p(f) \cdot p(g) \text {. }
$$

With the notation of $\S 2$ we have

LEMMA 3. $M^{K}$ is a multiplicatively locally convex algebra.

Theorem 2 follows from Lemma 2 and Lemma 3.

REMARK. The spectrum of the multiplicatively locally convex algebra $\left(H(U), \tau_{\omega}\right)$ can be used to give a construction of the envelope of holomorphy of $U$; see Matos [8]. For similar constructions with other devices see Alexander [1], Coeuré [4] and Schottenloher [12].

\section{REFERENCES}

1. H. Alexander, Analytic functions on Banach spaces, Thesis, University of California, Berkeley, Calif., 1968.

2. R. Aron, Holomorphy types for open subsets of a Banach space, Studia Math. 45 (1973), 273-289.

3. S. B. Chae, Holomorphic germs on Banach spaces, Ann. Inst. Fourier (Grenoble) 21 (1971), 107-141.

4. G. Coeuré, Fonctions plurisousharmoniques sur les espaces vectoriels topologiques et applications à l'étude des fonctions analytiques, Ann. Inst. Fourier (Grenoble) 20 (1970), fasc. 1, 361-432. MR 43 \#564.

5. S. Dineen, Topologie de Nachbin et prolongement analytique en dimension infinie, C. R. Acad. Sci. Paris Sér. A-B 271 (1970), A643-A644. MR 43 \#887.

6. - Holomorphy types on a Banach space, Studia Math. 39 (1971), 241-288. MR 46 \#3837.

7. - Holomorphic functions on $\left(c_{0}, X_{b}\right)$-modules, Math. Ann. 196 (1972), 106116. MR 45 \#9118.

8. M. Matos, Holomorphic mappings and domains of holomorphy, Centro Brasileiro de Pesquisas Fisicas, Rio de Janeiro, 1970.

9. J. Mujica, Spaces of germs of holomorphic functions, Thesis, University of Rochester, Rochester, N. Y., 1974.

10. L. Nachbin, Topology on spaces of holomorphic mappings, Ergebnisse der Mathe- 
matik und ihrer Grenzgebiete, Band 47, Springer-Verlag, New York, 1969. MR 40 \#7787.

11. L. Nachbin, Concerning spaces of holomorphic mappings, Rutgers University, New Brunswick, N. J., 1970.

12. M. Schottenloher, Über analytische Fortsetzung in Banachräumen, Math. Ann. 199 (1972), 313-336.

DEPARTMENT OF MATHEMATICS, UNIVERSITY OF ROCHESTER, ROCHESTER, NEW YORK 14627

Current address: Instituto de Matemática, Universidad Católica de Chile, Casilla 114-D, Santiago, Chile 